\title{
A study on Surgical Site Infections (SSI) and associated factors in a government tertiary care teaching hospital in Mysore, Karnataka
}

$\pi$
0
0
$\frac{1}{4}$
$\frac{1}{2}$

Naveen Kikkeri Hanumantha Setty, Manjunatha Shimoga Nagaraja,

Dinesh Halumatthigatta Nagappa', Chandrakumar Seegekote Giriyaiah, Naveen Ramanagaram Gowda ${ }^{2}$, Revathi Devi Mysore Laxmipathy Naik ${ }^{3}$

Departments of Community Medicine, ${ }^{1}$ General Surgery, ${ }^{3}$ Physiology, Mysore Medical College and Research Institute, Mysore, ${ }^{2}$ Department of Psychiatry,NIMHANS, Bangalore, Karnataka, India

Address for the Correspondence: Dr. Naveen K. H. D. no. 335, 'D' Subbaiaha Road, Behind Hotel Maharaja, Chamaraja Mohalla, Mysore - 570 024, Karnataka, India. E-mail: naveenkhdr@rediffmail.com

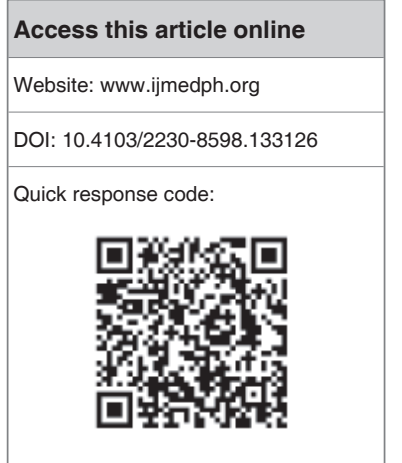

Background:Surgical site infections (SSI) are the most common nosocomial infection and frequently cause morbidity and mortality among inpatients of hospitals. The incidence varies from hospital to hospital. Several factors affect the development of SSI. Objectives:To study the incidence of and to identify the risk factors for Surgical Site Infections in the surgical wards of K.R.Hospital, Mysore. Materials and Methods: Study Period: July to August 2007 Study Subjects: One hundred and eighty patients who underwent various surgeries in the General Surgery department of K.R. Hospital Mysore. A predesigned and pretested proforma was used to collect the data. Surgical sites were examined and graded. Culture and sensitivity testing was done on infected wounds. Data was analyzed using SPSS 13 software. Results:Among 180 patients 39 (21.66\%) developed surgical site infections (SSI). Of 39 SSIs 20 were grade 3 and 19 were grade 4 infections. SSIs were found more commonly among the aged, males, underweight and overweight, anaemics, diabetics, hypertensives, patients with longer pre operative waiting time, with multiple blood transfusions and without antibiotic prophylaxis. Age, Sex, BMI, Diabetes mellitus, Blood transfusion and pre operative waiting had univariate statistical significance. Gender, extreme BMI, Diabetes mellitus and Blood transfusion remained independent predictors of surgical site infection in multivariate analysis. Most of the SSIs yielded multiple organisms and Staphylococcus aureus was the predominant. Resistance to tetracycline was most common. Conclusion:The incidence of SSI is high. Gender, extremes of BMI, diabetes mellitus and blood transfusion are the important risk factors for it.

Key words: K.R Hospital, Mysore, risk factors, surgical site infections

\section{INTRODUCTION}

Surgical site infections (SSI) frequently cause morbidity and mortality among inpatients of hospitals. They account for a considerable proportion of nosocomial infections among hospital inpatients. Hence they can act as surrogate markers for nosocomial infections. For surgical patients, SSIs are the most common nosocomial infection and they have been shown to be the leading cause of operation-related adverse events. ${ }^{[1,2]}$ Several studies have demonstrated an increased length of hospitalization and the associated financial implications for patients with SSI compared with non infected patients having similar surgical procedures. ${ }^{[3-5]}$ The prevalence varies from hospital to hospital and across different countries. ${ }^{[6-8]}$ When surgical patients with nosocomial SSI died, $77 \%$ of the deaths were reported as related to the infection and the majority $(93 \%)$ were serious infections involving organs or spaces accessed during the operation..$^{[9]}$ An estimate showed that if a hospital with an annual surgical volume of 10000 operations could reduce their SSIs by half; this would result in an average annual cost savings of approximately $\$ 450,000 \cdot .^{[10]}$

Total quality management in hospitals is gaining emphasis these days. Control of postoperative complications is an essential component of total quality management. In this context it becomes important to determine the prevalence of surgical site infections, assess the magnitude of the problem and provide a rationale to set priorities in infection control in the hospitals. Not many studies are done in India in this direction. Hence the present study had been undertaken. 


\section{AIM AND OBJECTIVES}

1. To study the incidence of Surgical Site Infections in the surgical wards of K.R.Hospital, Mysore.

2. To identify the risk factors for the development of SSIs.

3. To prepare an antibiogram to evaluate sensitivity pattern of organisms causing SSIs.

\section{MATERIALS AND METHODS}

\section{Study subjects}

One hundred and eighty patients who underwent various surgeries in the General Surgery department of K.R. Hospital Mysore were studied.

\section{Study setting}

K.R. Hospital which is a government tertiary care teaching hospital attached to Mysore Medical College and Research Institute, Mysore.

\section{Study period}

July-August 2007

\section{Inclusion criteria}

- Elective surgeries

- Those who stayed for at least seven days post-operatively.

\section{Exclusion criteria}

- Grossly contaminated or infected wounds/procedures

Following the surgical procedure, surgical sites were examined on postoperative day 3 and every 3 days thereafter. Wounds were graded on the following scale: grade $1=$ normal healing; grade $2=$ suture line erythema $<1 \mathrm{~cm}$; grade $3=$ suture line erythema $>1 \mathrm{~cm}$; grade $4=$ frank, purulent drainage. ${ }^{[1]]}$ Four surgeons did the grading for all the subjects. Cultures were obtained on all wounds determined to be infected or as otherwise clinically indicated. Grade 3 or 4 wounds were considered infected. Wounds from which a positive culture was obtained in the setting of physical signs of infection (i.e., fever, inflammation) were also considered infected. Antibiogram was prepared from culture sensitivity reports to evaluate the sensitivity pattern of organisms.

Demographic characteristics like age and sex were noted. Variables like BMI, co-morbid conditions, prophylactic antibiotic use, blood transfusion, and preoperative waiting period were compared in the infected and non-infected groups.

Statistical analysis was done using Microsoft Excel, SPSS 13 software. Those risk factors that were univariately significant using chi-square analysis at $p<0.05$ were entered into binary logistic regression equation to evaluate the risk of each factor when adjusted for other factors.

\section{RESULTS}

During the study period 180 patients underwent various surgeries in the general surgery department of K.R. Hospital, Mysore. Among them abdominal surgeries constituted majority (76.11\%) followed by limb surgeries. Others category included thyroid surgeries, lipoma and other tumor excisions [Table 1].

Among the 180 patients 39 developed surgical site infections giving a cumulative incidence of $21.66 \%$. Out of 39 SSIs 20 were grade 3 infections and responded to change in antibiotic guided by antibiogram. 19 patients developed grade 4 infection and some of them had constitutional symptoms like fever.

The age of study subjects ranged between 18 years to 67 years. Majority (64.44\%) of them belonged to $18-30$ years group. Out of 116 patients in this group $11(9.5 \%)$ got infected.19 patients were more than 50 years and among them 12(63\%) developed surgical site infection. It was found that the frequency of SSI increased with age and this was statistically significant [Table 2].

There were $110(61.11 \%)$ males and among them 32(29\%) got infected. Among the 70 women $7(10 \%)$ developed SSI. This difference in incidence was statistically significant. Majority of the study subjects (133-73.89\%) had normal BMI. Among them 10 (7.5\%) developed SSI. Out of 24 underweight patients $18(75 \%)$ developed SSI. Of the 23 overweight patients 11(47.83\%) developed infection of their surgical sites. This difference was found to be statistically significant [Table 2].

Anemia, hypertension and diabetes mellitus were the three comorbid conditions studied. Hemoglobin of 13 and $12 \mathrm{gm} \%$ were considered as the cut off points for the diagnosis of anemia in men and women respectively. Those with less than $10 \mathrm{gm} \%$ were considered severely anemic and these were the ones who received maximum blood transfusions. Among 37 anaemic patients who underwent surgery 23 (62.16) developed SSI.

The study subjects who knew their diabetic and hypertensive status before admission and those diagnosed after admission were considered diabetics and hypertensive respectively.

Majority of them knew their status before admission and majority were not taking medication regularly. This was one of the factors for increasing their preoperative waiting period. It was also found that majority had both conditions. Twenty $(83.33 \%)$ patients of the 24 diabetics and $17(73.91 \%$ ) of the 23 hypertensives respectively developed SSIs [Table 2].

Among the 180 study subjects $41(22.78 \%)$ patients received blood transfusions. Per operative transfusions accounted for major share of these transfusions. Eight $(5.7 \%)$ patients who did not receive any transfusion developed SSI. Four patients

\begin{tabular}{lc} 
Table 1: Distribution of surgeries according to site \\
\hline Surgical site & No. $(\%)$ \\
\hline Abdominal & $137(76.11)$ \\
Limbs & $29(16.11)$ \\
Others & $14(7.78)$ \\
Total & $180(100)$ \\
\hline
\end{tabular}




\begin{tabular}{|c|c|c|c|c|c|c|}
\hline \multirow[t]{2}{*}{ Factors } & & \multirow{2}{*}{$\begin{array}{c}\text { SSI } \\
\text { No. (\%) }\end{array}$} & \multirow{2}{*}{$\begin{array}{l}\text { No SSI } \\
\text { No. (\%) }\end{array}$} & \multirow{2}{*}{$\begin{array}{c}\text { Univariate } \\
P \text { value }\end{array}$} & \multicolumn{2}{|c|}{ Multivariate } \\
\hline & & & & & OR $(95 \% \mathrm{Cl})$ & $P$ value \\
\hline \multicolumn{7}{|l|}{ Age group (yrs) } \\
\hline $18-30$ & & $11(9.48)$ & $105(90.52)$ & \multirow{4}{*}{$<0.000$} & 1.00 & \\
\hline $31-40$ & & $12(32.4)$ & $25(67.6)$ & & $1.06(0.70-1.96)$ & 0.66 \\
\hline $41-50$ & & $4(50)$ & $4(50)$ & & $1.02(0.33-2.80)$ & 0.30 \\
\hline$>50$ & & $12(63.15)$ & $7(36.84)$ & & $1.26(0.89-1.80)$ & 0.87 \\
\hline \multicolumn{7}{|l|}{ Gender } \\
\hline Male & & $32(29.1)$ & $78(70.91)$ & \multirow{2}{*}{0.001} & 1.00 & \\
\hline Female & & $7(10)$ & $63(90)$ & & $0.43(0.31-0.73)$ & 0.003 \\
\hline \multicolumn{7}{|c|}{ Body Mass Index (BMI) } \\
\hline$<18.5$ & & $18(75)$ & $06(25)$ & \multirow{3}{*}{$<0.000$} & 1.00 & \\
\hline $18.5-25$ & & $10(7.52)$ & $123(92.48)$ & & $0.37(0.26-0.48)$ & 0.006 \\
\hline$>25$ & & $11(47.83)$ & $12(52.17)$ & & $1.56(1.39-2.65)$ & 0.02 \\
\hline \multicolumn{7}{|c|}{ Co morbid conditions* } \\
\hline \multirow[t]{2}{*}{ Anaemia } & Yes & $23(62.16)$ & $14(37.84)$ & \multirow{2}{*}{$<0.000$} & $1.25(0.73-1.81)$ & 0.98 \\
\hline & No & $16(11.19)$ & $127(88.81)$ & & 1.00 & \\
\hline \multirow[t]{2}{*}{ Hypertension } & Yes & $17(73.91)$ & $06(26.09)$ & \multirow{2}{*}{$<0.000$} & $1.13(0.69-1.92)$ & 0.54 \\
\hline & No & $22(14.01)$ & 135 (85.99) & & 1.00 & \\
\hline \multirow[t]{2}{*}{ Diabetes mellitus } & Yes & $20(83.33)$ & $04(16.67)$ & \multirow{2}{*}{$<0.000$} & $1.23(1.16-3.09)$ & $<0.000$ \\
\hline & No & $19(12.18)$ & $137(87.82)$ & & 1.00 & \\
\hline \multicolumn{7}{|l|}{ Blood transfusion } \\
\hline No transfusion & & $8(5.7)$ & $131(94.24)$ & \multirow{3}{*}{$<0.000$} & 1.00 & \\
\hline $1-3$ units & & $27(72.97)$ & $10(27.03)$ & & $2.02(1.77-4.80)$ & 0.004 \\
\hline$>3$ units & & $4(100)$ & $00(00)$ & & $6.26(2.89-9.08)$ & 0.02 \\
\hline \multicolumn{7}{|c|}{ Pre operative waiting period } \\
\hline$<2$ days & & $11(9)$ & $111(91)$ & \multirow{3}{*}{$<0.000$} & 1.00 & \\
\hline 2-7 days & & $16(53.33)$ & $14(46.67)$ & & $1.06(0.73-1.87)$ & 0.21 \\
\hline$>7$ days & & $12(42.86)$ & $16(57.14)$ & & $1.16(0.66-1.91)$ & 0.43 \\
\hline \multicolumn{7}{|l|}{ Prophylactic antibiotic } \\
\hline Yes & & $01(4.55)$ & $21(95.45)$ & \multirow{2}{*}{0.0375} & 1.0 & \\
\hline No & & $38(24.1)$ & $120(75.95)$ & & $1.08(0.82-1.17)$ & 0.41 \\
\hline
\end{tabular}

*Some SSIs were associated with multiple co-morbid conditions

received more than 3 units of blood and all of them developed infections [Table 2].

Majority (122-67.78\%) of the patients were operated within $48 \mathrm{~h}$ of admission and among them 11(9\%) developed SSI. Twenty eight $(15.56 \%)$ waited for more than a week to undergo surgery and among them $12(43 \%)$ developed SSI. The difference was statistically significant [Table 2].

It was observed that prophylactic antibiotic usage was not a routine in the studied hospital. Only selected patients who had some infection or other risk factor received antibiotic prophylaxis. Out of 22 patients who received prophylaxis only one person developed SSI. Of the remaining 158 patients, 38 (24\%) developed SSIs [Table 2].

The parameters which had univariate statistical significance were; Age, gender, BMI, Co morbid conditions: Anemia, Hypertentsion and Diabetes mellitus, Blood transfusion, pre operative waiting and prophylactic antibiotic usage. These were entered into multivariate analysis. Gender, Extreme BMI, Diabetes mellitus and Blood transfusion remained independent predictors of surgical site infection in multivariate analysis. Anemia, Hypertension and Prophylactic antibiotic usage did not show statistical significance [Table 2].

Among the 39 SSIs, 36 showed growth of colonies. Staphylococcus aureus was the predominant organism isolated from the surgical sites followed by Pseudomonas and Klebsiella. Some surgical sites had mixed infections involving multiple organisms [Table 3].

Organisms isolated from the SSIs showed resistance to all groups of antibiotics used in the surgery department of our hospital. Tetracycline encountered majority of the resistance followed by erythromycin and ampicillin. However these three antibiotics are not regularly used in our hospital. The commonly used antibiotics like Amikacin, Cephalexin and Cefotaxime also encountered resistance and this is a matter of concern [Table 4].

Once a SSI developed the surgeons order for culture and sensitivity tests and the antibiotics are changed accordingly. Co-morbid conditions like diabetes and hypertension were also monitored strictly. Despite these measures only 26(66.66\%) SSIs 


\begin{tabular}{lcc} 
Table 3: Organisms associated with SSI \\
\hline Organism & Frequency at SSI* No. & $(\%)^{\#}$ \\
\hline Staph. Aureus & 20 & 55.56 \\
Pseudomonas & 13 & 36.11 \\
Klb. Pneumonia & 10 & 27.78 \\
Citrobacter & 6 & 16.67 \\
E.Coli & 6 & 16.67 \\
Proteus & 5 & 13.89 \\
\hline
\end{tabular}

*Some SSIs were associated with multiple organisms, " of the 39 SSI only 36 showed the growth.

\section{Table 4: Antibiogram}

\begin{tabular}{lcc}
\hline Antibiotic & No. of resistant SSIs & \\
\hline Tetracycline & 34 & $\%$ \\
Erythromycin & 26 & 94.45 \\
Ampicillin & 22 & 72.23 \\
Gentamycin & 19 & 61.11 \\
Amikacin & 20 & 52.78 \\
Cotrimoxazole & 19 & 55.56 \\
Cephalexin & 18 & 52.78 \\
Norfloxacin & 16 & 50.00 \\
Ciprofloxacin & 17 & 44.45 \\
Cefotaxime & 16 & 47.23 \\
\hline
\end{tabular}

*Some organisms are resistant to more than one antibiotic, \# of the 39 SSI only 36 showed the growth

were completely healed at the time of discharge. 11(28.21\%) patients went home with SSI still persisting. One patient had to be operated again to control SSI and one elderly patient succumbed to septicemia.

\section{DISCUSSION}

Post-operative wound infection still remains one of the most important causes of morbidity and is the most common nosocomial infection ${ }^{[2,12]}$ in surgically treated patients. The present study was carried out among 180 elective surgery cases in the government tertiary care teaching hospital at Mysore, Karnataka state. Majority of the surgeries were abdominal followed by limb, thyroid surgeries, lipoma and other tumor excisions.

The rate of SSI varies greatly worldwide and from hospital to hospital. The rate of SSI varies from $2.5 \%$ to $41.9 \%$ as per different studies. ${ }^{[4,6-9]}$ The incidence of SSI in the present study is $21.66 \%$ even though high, is in agreement with the various studies.

The rate of SSI increases with the increase in age. In the current study a higher proportion of SSI was found among the subjects older than 50 years. This is comparable to other studies. ${ }^{[8,12-15]}$ This is due to poor immune response, existing co morbidities in old patients and reduced compliance with treatment. ${ }^{[8]}$

In the present study a significant proportion of males developed SSI compared to females. In another study in Pune, there was a marginal preponderance of male patients developing SSI $(7.4 \%)$ over female patients with SSI (5.1\%). ${ }^{[16]}$ In Aligarh, females $(27 \%)$ showed preponderance of SSI than males $(18 \%) \cdot{ }^{[17]}$ However according to Berard $\mathrm{F}$ and Gandon $\mathrm{J}$ sex is not a pre determinant of the risk of SSI. ${ }^{[18]}$

In the present study, both underweight $(\mathrm{BMI}<18.5)$ and overweight (BMI>25.0) have been found to significantly influence the onset of SSI. Similar results were found by Ashby etal..$^{[5]}$ In a study, by Xue etal., and Giles etal., higher BMI was a significant predictor of SSI. ${ }^{[14,19]}$

Co morbid conditions like anemia, diabetes and hypertension were the significant risk factors for SSI. Diabetes remained significant predictor in multivariate anlaysis. National Academy of Science also reported higher rate of infection in patients with Diabetes mellitus which is similar to our study. ${ }^{[13]}$ Comparable results were found in various studies involving different surgical procedures. ${ }^{[14,19]}$

A dose-response relationship was noted in the association between blood transfusion and SSIs in the current study. Majority of the transfusions were intra or per operative. In a study by Tang et al., blood transfusion was found to be an important risk factor in determining SSI ${ }^{[20]}$ which is similar to the present study. Allogeneic blood transfusion induces immunosuppression and predisposes to postoperative infection. ${ }^{[20]}$

A prolonged pre operative hospital stay with exposure to hospital environment has been shown to increase the risk of SSI. ${ }^{[6]}$ Comparable findings were found in the present study. Anvikar A.R. and Lilani S.P. also reported higher rate of SSI in patients with prolonged preoperative hospital stay. ${ }^{[6,7]}$ Prolonged preoperative hospital stay leads to colonization with antimicrobial resistant micro organisms and itself directly affects patient's susceptibility to infection either by lowering host resistance or by providing increased opportunity for ultimate bacterial colonization. ${ }^{[8]}$

Pre-operative antibiotics are known to decrease incidence of SSI cases. ${ }^{[7,18,21]}$ Prophylactic antibiotic usage was not a routine in the studied hospital. Only selected patients who had some infection or other risk factor received antibiotic prophylaxis. When it was not used a large proportion (24\%) developed SSI compared to the situations where such prophylaxis was given.

Staphylococcus aureus was the predominant organism isolated from the surgical sites followed by Pseudomonas and Klebsiella in the present study. E.coli, citrobacter and proteus were the other organisms isolated from SSIs. Lilani et al., and Mahesh et al., also found pre-ponderance of Staphylococcus aureus and Pseudomonas in SSIs in their studies. ${ }^{[6,22]}$ Many studies have reported Staphylococcus aureus as the commonest isolate from the postoperative wound infection. ${ }^{[9,23,24]}$ Other organisms have shown varied preponderance in different studies. Staphylococcus aureus forms the bulk of the normal flora of skin and nails. ${ }^{[25]}$ Hence; it is the commonest organism found in most of the SSIs. The high incidence of gram-negative organisms in the postoperative wound infections can be attributed to be acquired from patient's normal endogenous micro flora. ${ }^{[2]}$ 


\section{CONCLUSION}

The incidence of SSI was high. Age, gender, BMI, Co morbid conditions like Anemia, Hypertension and Diabetes mellitus, Blood transfusion, pre operative waiting and prophylactic antibiotic usage were risk factors for SSI. However on multivariable analysis only gender, extreme BMI, Diabetes mellitus and Blood transfusion were significant predictors for SSIs. Staphylococcus aureus was the most common organism associated with SSI. Majority of the SSIs were resistant to multiple antibiotics.

\section{RECOMMENDATIONS}

- Surveillance mechanisms should be developed for the control of SSI.

- Institutional antibiotic policy should be developed.

- Quality control exercises should become a routine and Medical auditing should be done periodically.

- Training of Nursing staff, technicians in postoperative wards regarding maintenance of strict aseptic environment needs emphasis. Concepts of barrier nursing and task nursing can go a long way in tackling SSI.

- Protocols should be developed for preoperative workup to control risk factors causing SSI.

\section{Limitations of this study}

- Incidence of SSIs was measured using Morris criteria in this study and the same is compared with studies using different criteria for diagnosing SSIs.

- $\quad$ Since the study duration was 2 months, generalization cannot be done.

- Culture was done for only few bacteriae because of operational feasibility.

\section{Areas of future research in K.R Hospital}

Assessment of sterilization status of the operation theatre suites, surgical instruments, cleanliness and hygiene of the surgical wards, nursing, paramedical staff, and their knowledge, attitude and practice regarding SSIs.

\section{REFERENCES}

1. Watanabe A, Kohnoe S, Shimabukuro R, Yamanaka T, Iso $\mathrm{Y}$, Baba H, et al. Risk factors associated with surgical site infection in upper and lower gastrointestinal surgery. Surg Today2008;38:404-12.

2. Suljagić $V$, Jevtic M, Djordjevic B, Jovelic A. Surgical site infections in a tertiary health care center: Prospective cohort study. Surg Today 2010:40:763-71.

3. Urban JA. Cost analysis of surgical site infections. Surg Infect (Larchmt)2006;7(suppl 1):S19-22.

4. Reichman DE, Greenberg JA. Reducing Surgical Site Infections: A Review. Rev ObstetGynecol 2009;2:212-21.

5. Eriksen HM, Chugulu S, Kondo S, Lingaas E. Surgical-site infections at Kilimanjaro Christian Medical Center. JHosp Infect 2003;55:14-20.

6. Lilani SP, Jangale N, Chowdhary A, Daver GB. Surgical site infection in clean and clean-contaminated cases. Indian $\mathrm{J}$ Med Microbiol 2005;23:249-52.

7. Anvikar AR, Deshmukh AB, Karyakarte RP, Damle AS, Patwardhan NS, Malik AK, et al. A one year prospective study of 3280 surgical wounds. Indian J Med Microbiol1999;17:129-32.

8. Patel SM, Patel MH, Patel SD, Soni ST, Kinariwala DM, Vegad MM. Surgical site infections: Incidence and risk factors in a tertiary care hospital, Western India. Natl JCommunity Med 2012;3:193-6.

9. Mangram AJ, Horan TC, Pearson ML, Silver LC, Jarvis WR Guideline for prevention of surgical site infection, 1999. Hospital Infection Control Practices Advisory Committee. Infect Control HospEpidemiol1999;20:250-78.

10. Available from: http://www.chcanys.org/clientuploads/downloads/ Clinical_Conf_2007/TracksMay/SIPNorthwestCollaborativeHandbook. pdf [Last cited on 2013 May 14].

11. Morris CD, Sepkowitz K, Fonshell C, Margetson N, Eagan J, Miransky $\mathrm{J}$, et al. Prospective identification of risk factors for wound infection after lower extremity oncologic surgery. Ann SurgOncol 2003;10:778-82.

12. Desa LA, Sathe MJ. Factors influencing wound infection (a prospective study of 280 cases). JPostgrad Med1984;30:231-6.

13. National Academy of Science/ National Research Council. Post operative wound infections: Influence of ultraviolet irradiation of the operating room and of various other factor.Ann Surg1964;160(supp2):1-132.

14. Xue DQ, Qian C, Yang L, Wang XF. Risk factors for surgical site infections after breast surgery: A systematic review and meta-analysis. Eur $J$ SurgOncol2012;38:375-81.

15. Ashby E, Davies MJ, Wilson AP, Haddad FS. Age, ASA and BMI as risk factors for surgical site infection measured using ASEPSIS in trauma and orthopaedic surgery. J Bone Joint Surg Br 2012;94 (SUPP 4):58.

16. Varsha S, Saikat B, Upendra L. Surgical site infections: A one year prospective study in a tertiary care center. Int $\mathrm{J}$ Health Sci (Qassim) 2012;6:79-84.

17. Khan MA, Ansari MN, Bano S. Post operative wound infection. Indian JSurg1985;48:383-6.

18. Berard F, Gandon J. Factors influencing the incidence of wound infection Ann Surg 1964;160:32-81.

19. Giles KA, Hamdan AD, Pomposelli FB, Wyers MC, Siracuse JJ, Schermerhorn ML. Body mass index: Surgical site infections and mortality after lower extremity bypass from the National Surgical Quality Improvement Program 2005-2007. Ann VascSurg2010;24:48-56.

20. Tang R, Chen HH, Wang YL, Changchien CR, Chen JS, Hsu KC, et al. Risk factors for surgical site infection after elective resection of the colon and rectum: A single-center prospective study of 2,809 consecutive patients. Ann Surg 2001;234:181-9.

21. Wong ES. Surgical site infections. In: Mayhall CG, editor. Hospital epidemiology and infection control. $1^{\text {st }}$ ed. USA: Williams and Wilkins; 1996.p.154-74.

22. Mahesh CB, Shivakumar S, Suresh BS, Chidanand SP, Vishwanath Y. A prospective study of surgical site infections in a teaching hospital. J ClinDiagn Res2010;4:3114-9.

23. Prabhakar H, Arora S. A bacteriological study of wound infections. J Indian Med Assoc 1979;73(9and10):145-8.

24. Malik S, Gupta A, Singh KP, Agarwal J, Singh M. Antibiogram of Aerobic Bacterial Isolates from Post-operative Wound Infections at a Tertiary Care Hospital in India. J Infect Dis Antimicrob Agents 2011;28:45-52.

25. Isibor JO, Oseni A, Eyaufe A, Ahmadu T. Incidence of aerobic bacteria and Candida albicans in postoperative wound infections. Afr J Microbiol Res2008;2:288-91.

How to cite this article: Setty $\mathrm{NH}$, Nagaraja MS, Nagappa DH, Giriyaiah CS, Gowda NR, Laxmipathy Naik RD. A study on Surgical Site Infections (SSI) and associated factors in a government tertiary care teaching hospital in Mysore, Karnataka. Int $\mathrm{J}$ Med Public Health 2014;4:171-5.

Source of Support: Nil, Conflict of Interest: None declared. 